\title{
Progress on Design and Construction of a MuCool Coupling Solenoid Magnet
}

\author{
L. Wang, X. K. Liu, F. Y. Xu, S. Y. Li, H. Pan, H. Wu, X. L. Guo, S. X. Zheng, M. \\ A. Green Member IEEE, D. R. Li, S. P. Virostek, M. S. Zisman
}

\begin{abstract}
The MuCool program undertaken by the US Neutrino Factory and Muon Collider Collaboration is to study the behavior of muon ionization cooling channel components. A single superconducting coupling solenoid magnet is necessary to pursue the research and development work on the performance of high gradient, large size $R F$ cavities immersed in magnetic field, which is one of the main challenges in the practical realization of ionization cooling of muons. The MuCool coupling magnet is to be built using commercial copper based niobium titanium conductors and cooled by two cryo-coolers with each cooling capacity of $1.5 \mathrm{~W}$ at $4.2 \mathrm{~K}$. The solenoid magnet will be powered by using a single $300 \mathrm{~A}$ power supply through a single pair of binary leads that are designed to carry a maximum current of 210A. The magnet is to be passively protected by cold diodes and resistors across sections of the coil and by quench back from the $6061 \mathrm{Al}$ mandrel in order to lower the quench voltage and the hot spot temperature. The magnet is currently under construction. This paper presents the updated design and fabrication progress on the MuCool coupling magnet.
\end{abstract}

Index Terms-MuCool superconducting magnet, updated design, coil, fabrication progress

\section{INTRODUCTION}

$\mathrm{T}$ HE MuCool at Feimi National Accelerator laboratory in US is one research and development program undertaken by the US Neutrino Factory and Muon Collider Collaboration to study the behavior of muon ionization cooling channel components including RF cavities and hydrogen absorbers [1]. The RF cavity to be used for the Muon Ionization Cooling Experiment (MICE) can be tested in a magnetic field under conditions similar to those encountered in the MICE cooling channel at MuCool test area (MTA). A prototype $201 \mathrm{MHz}$ RF

Manuscript received xx October 2009. This work was supported by Funds of cryogenics and superconductivity technology innovation project under "985-2 Plan" of Harbin Institute of Technology, China and by the Office of Science, US Department of Energy under DOE contract DE-AC02-05CH11231.

L. Wang was with the Institute of Cryogenics and Superconductivity Technology, HIT, Harbin 150001, China. She is now with Shanghai Institute of Applied Physics, Shanghai 201204, China (phone: 86-21-33932552, e-mail: wangli icst@hit.edu.cn).

X. K. Liu, F. Y. Xu, H. Pan, H. Wu, X. L. Guo, S. X. Zheng are with the Institute of Cryogenics and Superconductivity Technology, Harbin Institute of Technology, Harbin 150001, China.

S. Y. Li is with Hilong University of Science and Technology, Harbin 150027, China (e-mail: lisuyan010@sina.com).

M. A. Green, D. R. Li, S. P. Virostek, M. S. Zisman are with Lawrence Berkeley National Laboratory, Berkeley, CA, 94720 USA (e-mail: magreen@lbl.gov). cavity has been developed and achieved $\sim 16 \mathrm{MV} / \mathrm{m}$ peak accelerating field without any external magnetic field at Fermilab. A coupling solenoid magnet identical to those for the MICE is necessary for the MuCool to study the acceleration field under the magnetic field and perform the $201 \mathrm{MHz}$ RF cavity breakdown and dark current experiment. The acceleration gradient of the RF cavities that can be achieved under the magnetic field is one of the important inputs in the optimization of neutrino factory and muon collider design [2].

The Institute of Cryogenics and Superconductivity Technology (ICST) at the Harbin Institute of Technology (HIT) has been undertaking the design, fabrication and cryo-test two coupling magnets for MICE and the third functionally identical magnet for the MUCOOL experiment since 2006 in collaboration with the Lawrence Berkeley National Laboratory in US. This paper presents the updated design and fabrication progress on the MuCool coupling magnet.

\section{Updated Design of MuCoOl Coupling MAGNET}

The MuCool coupling magnet mainly consists of the coil cold mass assembly and the cryostat assembly as shown in Fig. 1 . The coil cold mass assembly at $4.2 \mathrm{~K}$ comprises the coil winding pack and its case, cooling tubing and helium vessels, cold end bases of cold mass support assembly and quench protection assembly. The cryostat includes the cryo-coolers and cooling circuit, cold mass supports, heat shields and their supports, magnet current leads, vacuum chamber, and instrumentation. The preliminary engineering design of the MICE/MUCOOL coupling magnets was carried out by ICST/HIT in collaboration with Lawrence Berkeley National Laboratory since December, 2006 and was first internationally reviewed in May 2007. According to the experience gained form development of two test coils [3][4], the detailed design was updated quite a lot and internationally reviewed again in December 2008 [5]. The updated design on the coil cold mass assembly and its cooling is presented in detail in this paper.

\section{A. Basic Parameters of MuCool Coupling Coi}

The updated design for the coil cold mass assembly is quite different from the preliminary one in terms of coil winding, mandrel fabrication, cooling and quench protection assembly [5]. Table I lists the basic parameters for the MuCool coupling coil. In practice, because the thinner fiberglass cloth with $0.050 \mathrm{~mm}$ thickness is to be used for the MuCool coil instead of $0.100 \mathrm{~mm}$ thick one, the coil thickness reduces from 
$110.4 \mathrm{~mm}$ to $105.5 \mathrm{~mm}$ considering the thickness of impregnating epoxy. Because the additional G-10 sheets are to be applied as the slip planes between the coil and the two mandrel end plates, the coil length becomes $284 \mathrm{~mm}$ instead of $285 \mathrm{~mm}$. The Catalyst 24LV with lower viscosity for curing the black Stycast epoxy is to be applied in the coil, so the turn-to-turn thickness may be controlled within $0.040 \sim 0.050$ $\mathrm{mm}$ during winding with improved wire alignment control, and then the coil turn per layer can be up to 168 turns. Consequently, the self-inductance and magnetic stored energy of the MuCool coupling coil is increased by $2.62 \%$ compared with the design parameters [6]. The peak magnet field is increased by $\sim 0.15 \mathrm{~T}$ at full current $210 \mathrm{~A}$. The coil temperature margin is reduced by $\sim 0.1 \mathrm{~K}$ due to the increase of peak magnetic field and stored energy.



Fig.1. 3D view of MuCool coupling magnet

TABLE I. BASIC PARAMETERS OF COUPLING COIL

\begin{tabular}{lll}
\hline \hline \multicolumn{1}{c}{ Parameter } & Previous & \multicolumn{1}{c}{ Updated } \\
\hline Coil Length (mm) & 285 & $285(284)^{*}$ \\
Coil Inner Radius (mm) & 744 & 750 \\
Coil Thickness (mm) & 102.5 & $110.4(105.5)^{*}$ \\
Number of Layers & 96 & 96 \\
No. Turns per Layer & 166 & 166 \\
Magnet Self Inductance (H) & $\sim 564$ & $\sim 591.8(607.3)^{*}$ \\
Magnet Jc (A mm-2) & 114.6 & $106.85(112.03) *$ \\
Magnet Current (A) & 210 & 210 \\
Magnet Stored Energy (MJ) & 12.4 & $13.1(13.4) *$ \\
Peak Induction in Coil (T) & 7.44 & $7.32(7.49) *$ \\
Coil Temperature Margin (K) & $\sim 0.8$ & $\sim 0.86(\sim 0.76) *$ \\
\hline \hline
\end{tabular}

* The practical value of the MuCool coupling coil under construction

\section{B. Coil Assembly and Its Cooling}

The coil assembly consists of the coil and the coil case as shown in Fig. 2. The coil is composed of electrical insulations, epoxy resin, and conductors made of copper based $\mathrm{NbTi}$ conductors with insulated dimension of $1.65 \mathrm{~mm}$ in width and
$1.00 \mathrm{~mm}$ in height. The coil case includes the mandrel, the cover plate and the banding. The coil mandrel made of 6061T6 aluminum consists of the inner bobbin and two end plates. The inner diameter of the coil bobbin is $1460 \mathrm{~mm}$, and its thickness is $19 \mathrm{~mm}$. The thickness of the end plate is 18 $\mathrm{mm}$, and the outer diameter of the left plate is $1860 \mathrm{~mm}$, that of the right plate is $1770 \mathrm{~mm}$. The cover plate also made from $6061 \mathrm{~T} 6 \mathrm{Al}$ is to be welded onto the coil mandrel after the banding is wound outside the $1.0 \mathrm{~mm}$ thick G-10 insulation sheet attached to the outermost layer of the coil. The length of the mandrel is $328 \mathrm{~mm}$. Compared with the previous design, the bobbin thickness increases from $13 \mathrm{~mm}$ to $19 \mathrm{~mm}$ considering the severe peak stress generated during cool down process in the bobbin center [8], which leads to tighter space between the bobbin and the inner vacuum chamber. The thickness of the cover plate increases from $15 \mathrm{~mm}$ to $45 \mathrm{~mm}$ because the helium cooling tubing is to be welded into the slots in it instead of extruded tubes attached onto its outer surface. Having the cooling tubes embedded into the cover plate improves the heat transfer from the coil to the liquid $\mathrm{He}$ tubes and provides more cold surfaces for easy mounting of quench protection assembly. The thicker cover plate also allows the reaction forces in the cold mass supports to be carried across the coil cover through the clevis fixture. The thickness of the banding is $27 \mathrm{~mm}$ which is almost same as the previous design. The banding is not only used for reinforcing the coil winding but also for enlarging the space between the cover plate welds and the conductors to protect the conductor formvar insulation from burning out during the welding process [5].

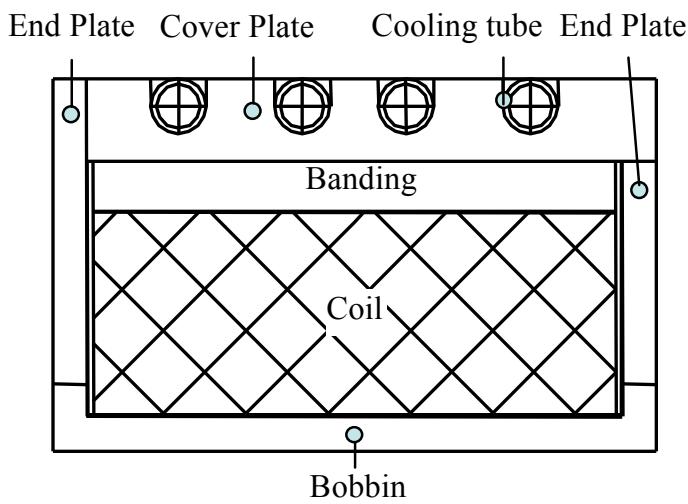

Fig. 2. Cross section of the MuCool coupling coil assembly

As shown in Fig. 3, the coil cooling circuit is to be arranged as a thermal siphon cooling system driven by gravity force to minimize the temperature drop between the second stage cold head of cooler and the hot spot in the magnet. The coupling coil cooling system mainly consists of cryocooler and helium re-condenser, piping system for various operations, liquid helium vessels and safety device. For each coupling solenoid, two PTR cryocoolers with $1.5 \mathrm{~W}$ cooling capacity at $4.2 \mathrm{~K}$ each must be used to provide the cryogen-free refrigeration in terms of heat loads in the magnet and effect of stray magnetic field on the cooler during normal operation. The combined 
refrigeration power will be $3.0 \mathrm{~W}$ at $4.2 \mathrm{~K}$ and $110 \mathrm{~W}$ at $60 \mathrm{~K}$ $(50 \mathrm{~Hz})$ or $90 \mathrm{~W}$ at $50 \mathrm{~K}$.

The PTR coolers will operate with the cold heads down and be mounted on top of the cryostat vacuum chamber using drop-in mode. The drop-in cooler is isolated from the cryostat vacuum by thin-wall helium-filled stainless steel sleeve. This arrangement allows for the easy removal of the cooler from the magnet cryostat in order to avoid potential damage during long-term transportation from China to USA or from USA to UK. The re-condenser is made from the holes in the copper block that comprises the second-stage of the cooler. The cooling capacity of the PTR-415 in the drop-in configuration was measured in USA, and only $1.2 \mathrm{~W} \sim 1.3 \mathrm{~W}$ was obtained probably due to the conduction through the thin-wall SS sleeve, which means the effective available cooling power at $4.2 \mathrm{~K}$ is from $2.4 \mathrm{~W}$ to $2.6 \mathrm{~W}$ instead of $3.0 \mathrm{~W}$. The estimated heat loads for the magnet are listed in Table II.
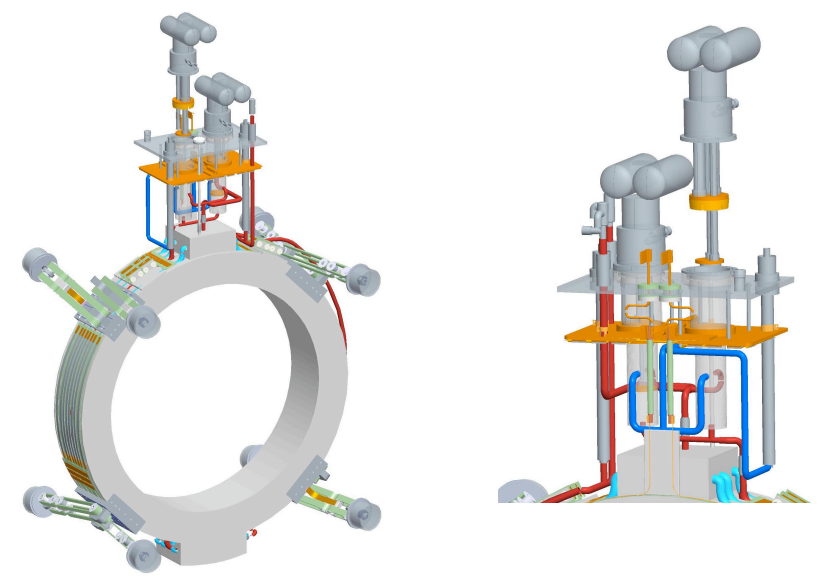

Fig. 3. Cooling circuit for the MuCool coupling magnet

TABLE II. HEAT LOADS AT $4.2 \mathrm{~K}$ (W)

\begin{tabular}{cc}
\hline \hline HTS leads (60K-4.2K) & 0.13 \\
Conduction through 4.2 K cold mass supports & 0.2 \\
Radiation to 4.2 K cold mass & 0.85 \\
$4.2 \mathrm{~K}$ neck tubes & 0.212 \\
SC joints & $2.64 \times 10^{\wedge}-3$ \\
$4.2 \mathrm{~K}$ instrument wires & 0.1 \\
Sub-total & 1.492 \\
Contingence & $30 \%$ \\
\hline \hline
\end{tabular}

\section{Quench Protection Assembly}

The MuCool coupling magnet will be passively quench-protected by sub-dividing the coil into eight parts. Each coil sub-division will have a diode and resistor across it.. Quench back from the coil mandrel and the banding in combination with the coil sub-division ensures that the hot spot temperature in the coil will be no higher than $120 \mathrm{~K}$ [7].

The quench protection assembly will be mounted on the outer surface of the coil cover plate and is kept cooled lower than $4.8 \mathrm{~K}$ by heat conduction during normal operation as shown in Fig. 4. It is divided into two groups, and each group is spread at one side of the helium vessels, which is composed of a cold diode assembly and a resistor assembly [5].

Each cold diode assembly is composed of 4 pairs of back-to-back diodes as shown in Fig. 4, which are R620 "hockey puck" high current rectifiers with industry grade. This kind of diode has been successfully applied in the cryogenic temperature. The forward voltage of the quench diode is very sensitive to the diode temperature, external magnetic field and its direction. The field around the diode is about $1.5 \mathrm{~T} \sim 2.5 \mathrm{~T}$. Before mounting, the diodes should be tested at low temperature (e.g. 4.2K) and magnetic field for verifying the quality and measuring the forward threshold voltage drop, forward and reverse leak current.

The diode assembly is designed as a sandwich-like structure in order to meet the demands on heat conduction, electrical conduction and elec-insulation simultaneously. Each pair of diodes is clamped between 2 copper plates with thickness of 4 $\mathrm{mm}$, which are used as both thermal sink and electrical connection for cold diodes. The G-10 sheets with thickness of $1 \mathrm{~mm}$ are used for electrical insulation among diode pairs and copper pieces. A stack of Bellville washers are to be used to maintain the clamping force with pressure of 1000-1400 lbs even at $4 \mathrm{~K}$ so as to keep good electrical contact of diode assemblies. The Al plates are used as fixture as well as thermal sink and cooling conduction. The G-10 washers and G-10 tubes are used to insulate bolt assembly with copper plates. The $5 \mathrm{kV}$ Hi-pot test should be performed for the diode assembly. The clear path between copper poles and the aluminum plate will be more than $10 \mathrm{~mm}$.
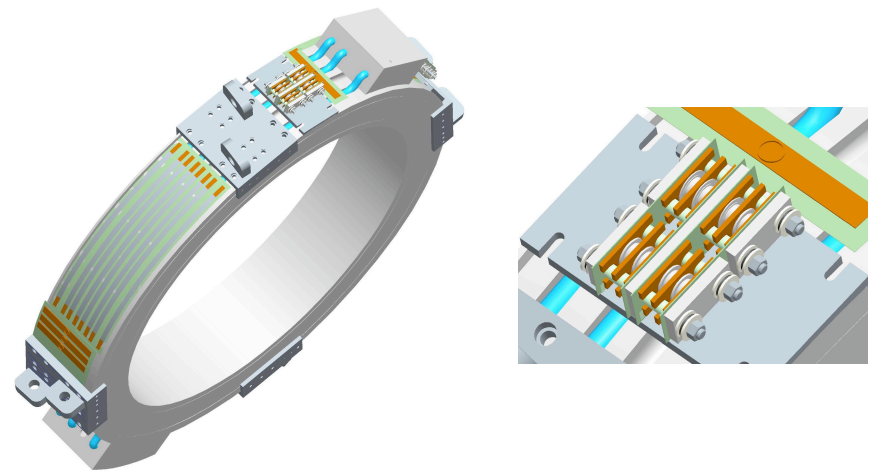

Fig. 4. Top view of passive quench protection assembly

According to the simulation results [5], for the 8-sectioned coupling coil with stainless steel banding during quench process, the hot spot temperate is about $130 \mathrm{~K}$, the voltage-to-ground is $2611 \mathrm{~V}$, the layer-to-layer voltage is 435 $\mathrm{V}$, and the quench time constant is about $16 \mathrm{~s}$. Most of the storage energy is dumped into the coil itself and the energy dumped to the banding is negligible.

\section{FABRICATION PROGRESS OF COUPLING MAGNET}

At the early of 2009, the contract to fabricate the MuCool coupling magnet was awarded to QiHuan Mechanical \& 
Electronic Engineering Company in BeiJing by HIT after inspection of LBNL collaborators to QiHuan at the early of this March. The fabrication includes the coil winding. The winding system except the winding machine was transported to the QiHuan from the ICST. The system was re-assembled and debugged by the QiHuan under ICST's inspection. The winding technique developed by ICST was transferred to the QiHuan by winding a small coil with $350 \mathrm{~mm}$ inner diameter using the conductors by this July. All the materials for the coil winding provided by LBNL were shipped to the QiHuan at the end of this May. The tooling supporting the mandrel during the winding was fabricated. The coil winding will start once the test for the prototype coil is to be completed and the mandrel fabrication is to be done. The 304 stainless steel plates and the $4 \mathrm{~mm}$ thick 1100 -aluminum plates have been procured for the fabrication of the vacuum chamber and the heat shields for the magnet cryostat.

For the coil cold mass assembly, the helium vessels were ready for welding with the cooling tubing. The helium cooling tubing made from $6061 \mathrm{Al}$ with $30 \mathrm{~mm}$ outer diameter and 4 $\mathrm{mm}$ thickness has been leakage checked under the pressure 2.5 $\mathrm{MPa}$. The leakage rate is less than $1 \times 10^{\wedge}-10 \mathrm{~Pa} / \mathrm{m}^{\wedge} 3-\mathrm{s}$. The forged cover plate was machined to final dimensions.

The current 6061-T6 Al coil mandrel is fabricated from two cut end plates and a roll-up plate. The welding of the end plates onto the roll-up was done in a way to minimize distortion. Ten pass full penetration welds at each side were used on the mandrel. The mandrel distortion was measured at 24 places around the mandrel as it was welded and quite small. The width across the mandrel plates is in $\sim 333 \pm 0.7$ $\mathrm{mm}$.

After welding, the strength of thermal effect region around welds lowers down greatly. The material around welds will change to 6061-0 and its tension strength is only $\sim 125 \mathrm{MPa}$. Proper heat treatment is helpful to increase the strength around welds and to release the thermal stress generated during welding. The material around welds will change to 6061-T4 with tension strength of $\sim 207 \mathrm{MPa}$ or be back to 6061 -T6 with tension strength of $\sim 290 \mathrm{MPa}$. However, the heat treatment will lower down the electrical performance of material such as elec-conductance. The mandrel will be machined to its final dimensions after it is heat treated to strengthen the welds $(>200 \mathrm{MPa})$ as planned previously.

The key points for heat treatment include proper processing procedure and parameters (needed to be tested using samples) , annealing oven with precise temperature control system, stable and uniform temperature on parts, and limited quench time $(<20 \mathrm{~s})$. The heat treatment procedure have been studied and proved using small samples by the QiHuan for more than ten times from the middle of this May till the end of this July. However, it's hard to realize the processing on the MuCool coupling coil mandrel using current oven without much modification due to large size.

According to the suggestion from the experts both in China and in USA, there are two options, one is to develop one specialized salt bath oven for the MuCool coil mandrel heat treatment, the other is to machine one new mandrel from a single forged 6061-T6-aluminum cylinder instead. Considering both cost and schedule, the latter is preferred.

\section{CONCLUSION}

The updated design for the MuCool coupling magnet was carried out according to experience gained from development of two test coils by ICST and internationally reviewed in December, 2008. The MuCool coupling magnet is under fabrication. Extensive tests for mandrel welding and heat treatment process after welding have been carried out. According to the experimental results, the forging coil mandrel will be adopted instead of welding. The coil winding is expected to start at the early of 2010 .
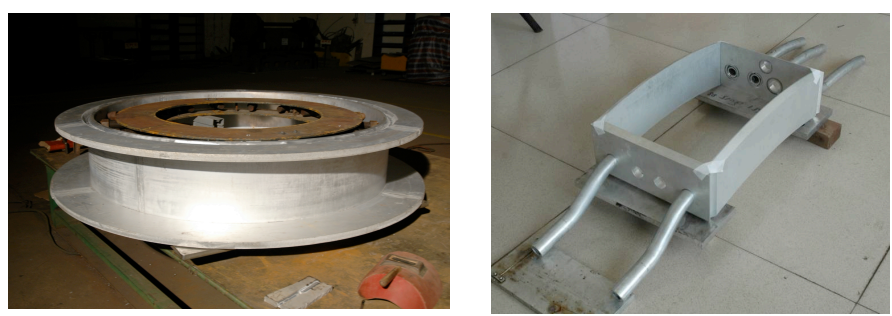

Fig.5. Mandrel after welding, pre-assembly of cooling tubing and He vesel

\section{ACKNOWLEDGMENT}

The authors would like to thank the experts CHEN Hao-shu and YI Chang-lian from Beijing China for their technical assistance.

\section{REFERENCES}

[1] D. Li and M. S. Zisman, "201-MHz NCFR Cavity Program," (at MUCOOL in Fermilab), Neutrino Factory and Muon Collider Collaboration Meeting (March 2006), [Online]. Available: http://www.mice.iit.edu/nfmcc06/

[2] G. Gregoire, G. Ryckewaert, L. Chevalier, et al, "MICE and International Muon Ionization Cooling Experiment Technical Reference Document," [Online]. Available: http://hep04.phys.itt.edu/cooldemo.

[3] L. Wang, H. Pan, F. Y. Xu, et al., "Design and Construction of Test Coils for MICE Coupling Solenoid Magnet", IEEE Transactions on Applied Superconductivity, Vol. 19, No.3, 1340-1343, 2009.

[4] L. Wang, X. L. Gou, et al., "Design and Construction of a Prototype Solenoid Coil for MICE Coupling Magnets", to be submitted to MT21, HeFei, Oct.18-23, 2009.

[5] Engineering design of MICE/MUCOOL coupling solenoid magnets, Institute of Cryogenics and Superconductivity Technology, Harbin Institute of Technology, 2008.

[6] L. Wang, F. Y. Xu, H. Wu, et al., "Magnetic and Cryogenic Design of MICE Coupling Solenoid Magnet System", IEEE Transactions on Applied Superconductivity, Vol.19, No.3, 1344-1347, 2009.

[7] X. L. Guo, F. Y. Xu, L. Wang, M. A. Green, et al., "Quench Protection for the MICE Cooling Channel Coupling Magnet", IEEE Transactions on Applied Superconductivity, Vol.19, No.3, 1360-1363, 2009 